\title{
SEPARATED INSTRUMENTS- A MIND-SET BETWEEN HARD AND ROCK- A REVIEW
}

\author{
Geeth Deepika1', Suresh Mitthra², Balasubramaniam Anuradha ${ }^{3}$, Arumugam Karthick ${ }^{4}$
}

1 Postgraduate Student, Department of Conservative Dentistry and Endodontics, Sree Balaji Dental College and Hospital.

${ }^{2}$ Reader, Department of Conservative Dentistry and Endodontics, Sree Balaji Dental College and Hospital.

${ }^{3}$ Reader, Department of Conservative Dentistry and Endodontics, Sree Balaji Dental College and Hospital.

${ }^{4}$ Associate Professor, Department of Conservative Dentistry and Endodontics, Sree Balaji Dental College and Hospital.

\section{ABSTRACT}

\section{BACKGROUND}

With an increased practice of rotary endodontics in recent years separated rotary nickel-titanium (NiTi) files in root canals is the most commonly reported mishap, causing lot of stress and anxiety among clinicians and patients. No clear guidelines can be drawn from the literature available, because there are either too few studies about the effects of broken files on prognosis or few studies that have been performed on patients. The prognosis is also dependent on file location, prior condition of the pulp, presence or absence of periapical lesion and many other factors. Intracanal separation of endodontic instruments may hinder cleaning and shaping procedures within the root canal system with a potential impact on the outcome of treatment. This article presents an overview of the literature regarding management of separated intracanal instruments.

\section{LITERATURE AND DATA SOURCES}

Pertinent literature search was carried out with the focus of management of separated instruments and its impact on treatment outcome and recent techniques used for retrieval. Search engines from PubMed and Google Scholar were used to identify relevant English language studies and literature published till the year 2017 and we have selected 50 articles using the following keywords: Fractured, instruments, nickel titanium, retrieval, bypassing, management. The scientific papers were then screened for their relevance to the intended objectives and were shortlisted to 30 articles. This review article presents an overview of the literature regarding management of separated intracanal instruments and its impact on treatment outcome.

\section{KEYWORDS}

Fractured, Instruments, Nickel Titanium, Retrieval, Bypassing, Management.

HOW TO CITE THIS ARTICLE: Deepika G, Mitthra S, Anuradha B, et al. Separated instruments- a mind-set between hard and rocka review. J. Evolution Med. Dent. Sci. 2017;6(87):6077-6080, DOI: 10.14260/jemds/2017/1319

\section{BACKGROUND}

The main objective of root canal treatment is complete debridement and uniform shaping of the root canal system. ${ }^{1}$ There are many procedural errors commonly faced by the clinician in a day-to-day endodontic practice. ${ }^{2}$ However, separated endodontic instruments serves as a stumbling block in achieving this objective. It is an unfortunate occurrence that may hinder root canal procedures and affect the treatment outcome. ${ }^{3}$ The term "broken instruments" applies not only to separated files, but it could also apply to a sectioned silver point, files, reamers, a segment of lentulo spiral, GG drill (gates glidden), lateral or finger spreaders. ${ }^{4}$ In order to overcome this, the composition and design of root canal instruments have been modified by the manufacturers for a better performance and fewer undesirable complications like instrument separation. Even besides the advent of Nickel-Titanium (NiTi) alloys have not resulted in a lower incidence of instrument separation, because it undergoes unexpected fracture without any signs of visible deformation. ${ }^{5}$ The incidence rate of instrument separation for stainless steel instrument ranges between $0.25 \%$ and $6 \%$,

'Financial or Other Competing Interest': None.

Submission 23-09-2017, Peer Review 18-10-2017,

Acceptance 24-10-2017, Published 30-10-2017.

Corresponding Author:

Dr. Geeth Deepika,

Department of Conservative Dentistry and Endodontics,

Sree Balaji Dental College and Hospital,

Chennai- 600100

E-mail: geethdeepika@gmail.com

DOI: $10.14260 /$ jemds/2017/1319 whereas for NiTi rotary instruments ranges from $1.3 \%$ to $10.0 \% .^{6,7}$ In spite of many factors that contribute to instrument separation, the exact mode of separation is not fully understood. This problem of instrument separation occurs even from experienced hands, which frustrates both the practitioners and patients. This article presents an overview of the impact of retained separated instruments on treatment outcome influencing factors, techniques and treatment options for management of separated instruments.

\section{Impact of Retained Separated Instruments on Treatment Outcome}

A separated instrument does not necessarily mean surgery or loss of the tooth. When an instrument separates into the root canal, it is important to address two main issues to maximise the treatment outcome. ${ }^{8}$ The first and foremost is the existence of a metal fragment inside the tooth and the possibility of corrosion. Corrosion mainly occurs with the use of silver points, whereas stainless steel and NiTi rotary instruments are inert in nature. ${ }^{9}$ The second concern is that the separated instrument blocks or hinders the access to the apical foramen and hence the objective of root canal treatment that is cleaning and shaping is compromised and impairs the treatment outcome. The most common cause of periapical lesion is intracanal infection. ${ }^{10}$ Some clinicians have experienced no clinical or diagnostic signs of periapical inflammation even in the presence of separated instrument, because of the fact that broken file does not induce inflammation by itself. Strindberg et al reported a $19 \%$ decrease in the rate of healing of periapical tissues when separated instruments were present. But the drawback of this study is small number of cases with pre-existing 
periapical lesion. ${ }^{11}$ Thus, a separated instrument itself does not predispose the case to post-treatment disease. The prognosis depends on 3 factors: What stage of instrumentation the separation occurred; Preoperative status of pulp and Periradicular tissues; Whether or not the file can be removed or bypassed. ${ }^{12}$ If the preoperative pulp was vital, an uninfected prognosis is better. In case if there is presence of necrotic and infected pulp and apical periodontitis, the prognosis will be uncertain. Panitvisai et al, a systematic review and meta-analysis concluded that the prognosis for fractured instrument fragment within a root canal is not significantly reduced. ${ }^{13}$ Thus, the presence of preoperative periapical radiolucency rather than the separated instrument per se is clinically more significant and demonstrates that it has a negative influence on treatment outcome.

\section{Management Options for Separated Instruments}

Management of separated instruments is always a clinical dilemma when an instrument fractures in the root canal system. A clinical decision has to be made to leave, bypass or remove the fragment. The choice is based on the assessment of the potential risks and benefits. In spite of alternative treatment options for the management of separated instruments, the optimum management is removal of the fragment in order to fulfil the objectives of root canal treatment like cleaning and shaping of the root canal system to effectively eliminate microorganisms. ${ }^{14}$

\section{Factors Influencing the Removal of Separated Instruments}

Some of the factors that aid in the successful retrieval of separated instruments are canal anatomy including its crosssectional shape, diameter, length and curvature of the canal. The likelihood of separation of instrument is the mandibular molars, mesial canal and in the mesiobuccal (MB) roots of maxillary molars. Because these roots not only curve distally, but often the MB canal curves lingually and the mesiolingual canal (ML) curves slightly to the buccal. These curvatures are not visible on an Intraoral periapical radiograph (IOPAR). Other factors include composition of the fractured fragment whether it is a stainless steel or NiTi, length of the fragment and its location in the coronal, middle or apical third of the canal or beyond apex, thickness of dentin and depth of an external concavity. 15

\section{Factors Governing the Successful Retrieval of an Instrument}

The two main factors governing the successful retrieval of an instrument are optics, coronal and radicular access. Operating under a high magnification dental operating microscope (DOM) improves vision and significantly increases the likelihood of retrieval. Coronal access a proper coronal flaring and SLA (Straight-Line Access) is the first and foremost step in removing the fragment. ${ }^{16}$ Radicular access after adequate coronal flaring and hand filing is done till the visible part of the fragment and Gates Glidden drills are used in a sequential manner to provide access and visibility. ${ }^{17}$

Techniques Used for Removing the Separated Instrument During the past several decades many devices, techniques and methods have been described for the removal of separated instruments. Some are still widely used, others are only of historical interest. ${ }^{18}$ Till date several new promising techniques and devices have emerged in market. But it is absolutely necessary that a clinician effectively and safely handle the devices and instruments that are used for removing the separated fragment to avoid further complications. Some of them are chemical solvents, mini forceps, broach and cotton, wire loops, hypodermic surgical needles, braiding of endodontic files, Masserann instruments, extractors, ruddle system, canal finder system, ultrasonics, file removal system, softened gutta-percha point and microtube method. ${ }^{19}$ The principles involved in the retrieval are tube-sleeve-fit principle. According to this technique, a microtube is placed over the exposed part of the fragment and a corresponding stylus is used to "lock" the fragment. An adhesive could also be used with the microtube. This technique is mainly indicated when the fragments are present in the coronal and middle third with adequate amount of dentin. ${ }^{20}$ The second principle is disengaging; rebound and recover in this Ultrasonic (US) vibrations are used to "tease" the fragment present coronally. This technique is indicated in apical third with the coronal most part of the fragment just beyond curvature or when minimal dentin exists peripheral to the fragment.

Ultrasonics is the most commonly used technique for retrieval of separated instruments with a success rate of $88 \%$ and $95 \%$, were reported in association with the operating microscope. ${ }^{21} \mathrm{~A}$ staging platform is prepared in the preenlarged canal with the help of modified GG (Gates Glidden drill) and ultrasonic tips are used to trephine dentin around the fragment. The vibration and energy from the ultrasonic tips helps to loosen and the separated fragment poops out of the canal. ${ }^{22}$ There are variety of techniques which are accomplished for trephining the dentin surrounding the fragment, some variants uses microtube. The disadvantages of the retrieval of separated files have been excessive removal of root dentin during trephine, which may result in perforation or predispose the teeth to vertical root fracture, especially in the apical third. ${ }^{23,24}$ Next most common device is Masserann kit (micro-mega). It consists of a traditional hollow cutting end tube with a diameter from $1.1-2.4 \mathrm{~mm}$ and requires only considerable amount of removal of dentin. ${ }^{19}$ Recently, a new small-diameter trepan bur system (Micro-Retrieve and Repair System; Superline NIC Dental, Shenzhen, China) with a diameter from $0.7-1.2 \mathrm{~mm}$ and thickness of $0.15 \mathrm{~mm}$ less than that of other devices was introduced. It consists of a trepan bur and microtubes. In order to improve the access, the bur and microtube length can be adjusted. When the fragment is exposed to a 1 to 1.5 $\mathrm{mm}$ length, then the microtube is used to withdraw the fragment. Because of its small-sized trepan bur technique, it is superior to ultrasound with regard to the amount of dentin removed and the time taken for removal of fractured instruments from root canals is faster. ${ }^{25}$

\section{Consequences of Fractured Instruments}

The fractured instruments will create unclean and unfilled spaces within the root canal system as well as unnecessary removal of excess dentin during removal procedure. There are four major complications like ledge formation, instruments used for removal may themselves separate and complicate treatment further, root perforation during preparation of staging platform, extrusion of the fragment 
apically because of excessive pressure application during the retrieval of separated instruments. These might further complicate the treatment outcome.

\section{Bypassing the Separated Instrument}

Bypassing a fragment located deep in the root canal or beyond the root canal curvature, it fulfils the objective of root canal treatment establishing a proper cleaning and shaping of the root canal system to some extent. ${ }^{26}$ Bypass is done by inserting a file between the fragment and root canal wall, thereby negotiating the canal to full working length. But it also creates a false channel parallel to the original root canal, which often leads to a root perforation. If a file is separated it is not advised to place another NiTi file to bypass the fractured instrument, because the odds of the second file to separate are very high and the treatment of bypassing the first becomes poor. It has also been reported that if the file is bypassed, the retained fragment does not compromise obturation quality. ${ }^{27}$

\section{Leaving the Fragment In Situ}

If a separated instrument cannot be removed or bypassed, then refer the patient to an experienced specialist. Otherwise, the alternative treatment option is cleaning and shaping till the level of the separated fragment. This is usually applicable for cases that are in final stages of root canal preparation or when the fragment is located beyond the curvature, i.e. apical third. ${ }^{28}$ From the patient's point of view retaining the fractured instruments creates anxiety, as it can be viewed as a treatment failure or even clinical negligence and may be perceived as the source of any problem the patient may encounter in the future. These patients should be frequently called for regular examination. In case there occurs a posttreatment disease, then surgical approach is the only option.

\section{CONCLUSION}

The decision on the management of separated instruments should consider the following like constraints of the root canal accommodating the fragment, the stage of root canal instrumentation at which the instrument armamentaria is available, possible complications, the strategic importance of the tooth involved and the presence or absence of periapical pathosis, understanding of these influencing factors as well as the ability to make a balanced decision are essential. Thus, the preventive measures include the case selection, experience of the clinician, limited re-use and then techniques used for retrieval of separated instruments.

\section{REFERENCES}

[1] Vertucci FJ. Root canal anatomy of the human permanent teeth. Oral Surg Oral Med Oral Pathol 1984;58(5):589-99.

[2] Yadav RK, Chand S, Verma P, et al. Clinical negligence or endodontic mishaps: a surgeons dilemma. Natl J Maxillofac Surg 2012;3(1): 87-90.

[3] Torabinejad M, McDonald NJ. Endodontic surgery. In: Torabinejad M, Walton RE. eds. Endodontics principles and practice. $4^{\text {th }}$ edn. St Louis: Elsevier Health Sciences 2009:357-75.
[4] Parashos P, Messer HH. Rotary NiTi instrument fracture and its consequences. J Endod 2006;32(11):1031-43.

[5] Pettiette MT, Conner D, Trope M. Procedural errors with the use of nickel-titatium rotary instruments in undergraduate endodontics. J Endod 2002;28:259.

[6] Al-Fouzan KS. Incidence of rotary ProFile instrument fracture and the potential for bypassing in vivo. Int Endod J 2003;36(12):864-7.

[7] Schafer E, Schulz-Bongert U, Tulus G. Comparison of hand stainless steel and nickel titanium rotary instrumentation: a clinical study. J Endod 2004;30(6):432-5.

[8] Spili P, Parashos P, Messer HH. The impact of instrument fracture on outcome of endodontic treatment. J Endod 2005;31(12):845-50.

[9] Eleazer PD. Lack of corrosion of stainless steel instruments in vivo by scanning electron microscope and microprobe analysis. J Endod 1991;17(7):346-9.

[10] Saunders JL, Eleazer PD, Zhang P, et al. Effect of a separated instrument on bacterial penetration of obturated root canals. J Endod 2004;30(3):177-9.

[11] Strindberg LZ. The dependence of the results of pulp therapy on certain factors. An analytical study based on radiographic and clinical follow-up examinations. Acta Odontol Scand: Mauritzon 1956;14:1-175.

[12] Crump MC, Natkin E. Relationship of a broken root canal instrument to endodontic case prognosis: a clinical investigation. J Am Dent 1970;80(6):1341-7.

[13] Panitvisai P, Parunnit P, Sathorn C, et al. Impact of a retained instrument on treatment outcome: a systematic review and meta-analysis. J Endod 2010;36(5):775-80.

[14] Schilder H. Filling root canals in three dimensions. Dent Clin North Am 1967:723-44.

[15] Hulsmann M, Schinkel I. Influence of several factors on the success or failure of removal of fractured instruments from the root canal. Endod Dent Traumatol 1999;15(6):252-8.

[16] Sjogren U, Hagglund B, Sundqvist G, et al. Factors affecting the long-term results of endodontic treatment. J Endod 1990;16(10):498-504.

[17] Suter B, Lussi A, Sequeira P. Probability of removing fractured instruments from root canals. Int Endo J 2005:38(2):112-23.

[18] Gencoglu N, Helvacioglu D. Comparison of the different techniques to remove fractured endodontic instruments from root canal systems. Eur J Dent 2009;3(2):90-5.

[19] Okiji T. Modified usage of the Masserann kit for removing intracanal broken instruments. J Endod 2003;29(7):466-7.

[20] Wei X, Ling JQ, Gao Y, et al. Management of intracanal separated instruments with the microsonic technique and its clinical outcome. Zhonghua Kou Qiang Yi Xue Za Zhi 2004;39(5):379-81.

[21] Fu M, Zhang Z, Hou B. Removal of broken files from root canals by using ultrasonic techniques combined with dental microscope: a retrospective analysis of treatment outcome. J Endod 2011;37(5):619-22. 
[22] Ruddle CJ. Nonsurgical retreatment. J Endod 2004;30(12):827-45.

[23] Souter NJ, Messer HH. Complications associated with fractured file removal using an ultrasonic technique. J Endod 2005;31(6):450-2.

[24] Madarati AA, Qualtrough AJ, Watts DC. Vertical fracture resistance of roots after ultrasonic removal of fractured instruments. Int Endod J 2010;43(5):424-9.

[25] Yang Q, Shen Y, Huang D, et al. Evaluation of two trephine techniques for removal of fractured rotary Nickel-titanium instruments from root canals. J Endod 2017;43(1):116-20.
[26] Nevares G, Cunha RS, Zuolo ML, et al. Success rates for removing or bypassing fractured instruments: a prospective clinical study. J Endod 2012;38(4):442-4.

[27] Radeva E. Bypassing a Broken Instruments. IJSR 2017;6(2):227-9.

[28] McGuigan MB, Louca C, Duncan HF. Clinical decisionmaking after endodontic instrument fracture. Br Dent J 2013;214(8):395-400. 